Article

\title{
Molecular Quantum Spintronics: Supramolecular Spin Valves Based on Single-Molecule Magnets and Carbon Nanotubes
}

\author{
Matias Urdampilleta ${ }^{1}$, Ngoc-Viet Nguyen ${ }^{1}$, Jean-Pierre Cleuziou ${ }^{1}$, Svetlana Klyatskaya ${ }^{2}$, \\ Mario Ruben ${ }^{2,3}$ and Wolfgang Wernsdorfer ${ }^{1, *}$ \\ 1 Institut Néel, CNRS et Université Joseph Fourier, BP 166, F-38042 Grenoble Cedex 9, France; \\ E-Mails: matias.urdampilleta@grenoble.cnrs.fr (M.U.); \\ ngoc-viet.nguyen@grenoble.cnrs.fr (N.-V.N.); jean-pierre.cleuziou@grenoble.cnrs.fr (J.-P.C.) \\ 2 Institute of Nanotechnology (INT), Karlsruhe Institute of Technology (KIT), \\ 76344 Eggenstein-Leopoldshafen, Germany; E-Mails: svetlana.klyatskaya@kit.edu (S.K.); \\ mario.ruben@kit.edu (M.R.) \\ 3 Institute de Physique et Chimie de Matériaux de Strasbourg (IPCMS), CNRS-Université de Strasbourg, \\ 67034 Strasbourg, France
}

* Author to whom correspondence should be addressed; E-Mail: wolfgang.wernsdorfer@grenoble.cnrs.fr; Tel.: +33-0-47688-7909.

Received: 18 July 2011; in revised form: 14 September 2011 / Accepted: 26 September 2011 / Published: 10 October 2011

\begin{abstract}
We built new hybrid devices consisting of chemical vapor deposition (CVD) grown carbon nanotube (CNT) transistors, decorated with $\mathrm{TbPc}_{2}(\mathrm{Pc}=$ phthalocyanine) rare-earth based single-molecule magnets (SMMs). The drafting was achieved by tailoring supramolecular $\pi-\pi$ interactions between CNTs and SMMs. The magnetoresistance hysteresis loop measurements revealed steep steps, which we can relate to the magnetization reversal of individual SMMs. Indeed, we established that the electronic transport properties of these devices depend strongly on the relative magnetization orientations of the grafted SMMs. The SMMs are playing the role of localized spin polarizer and analyzer on the CNT electronic conducting channel. As a result, we measured magneto-resistance ratios up to several hundred percent. We used this spin valve effect to confirm the strong uniaxial anisotropy and the superparamagnetic blocking temperature

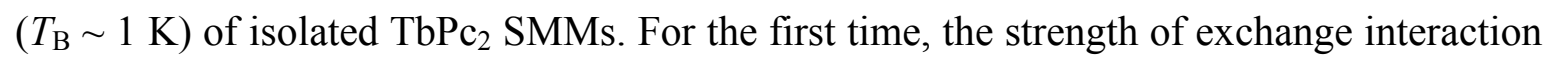
between the different SMMs of the molecular spin valve geometry could be determined.
\end{abstract}


Our results introduce a new design for operable molecular spintronic devices using the quantum effects of individual SMMs.

Keywords: molecular quantum spintronics; molecular magnets; nanoelectronics devices

\section{Introduction}

Single molecule magnets (SMMs) have attracted much interest over the last years because of their unique magnetic properties. These molecular structures combine the classical properties of magnets with the intrinsic quantum nature of nanoscale entities. With a large spin ground state and a magnetic anisotropy well-defined, molecular clusters composed of few magnetic atoms have shown various properties such as the blocking of the spin orientation at low temperatures, quantum tunneling of magnetization (QTM) [1] and interference effects between tunneling paths [2]. Besides, the synthetic chemistry produces controlled molecular structures at high yield and low cost. As a result, a wide range of SMMs systems incorporating transition metal and/or rare earth metal ions with tailored magnetic interactions have been discovered. In addition, the rich variety of quantum systems provided by the molecular magnetism field strongly motivates the use of SMMs for both quantum information storage and processing purposes.

At the same time, studies in spintronics using magnetic materials in electronic devices have made considerable progress from fundamental studies to practical applications. This technology is based on the discovery of magnetoresistive effects, such as the giant magnetoresistance effect, where metallic spin valves are composed of two metallic layers separated by a non-magnetic one. Depending on the relative magnetization orientation of the two magnets (parallel or antiparallel), a drastic change of the electrical resistance is observed. Nowadays, new directions in spintronics aim at transposing the existing concepts and at developing alternative ones with various types of materials, from inorganic to $\pi$-conjugated organic semiconductors [3]. Organic semiconductors are promising since they may offer longer spin relaxation times [4] than conventional transition metals as well as new functionalities (e.g., switchability with light, electric field, etc.). In this context, SMMs are interesting candidates to study and preserve quantum coherence of the electronic spin in molecular spintronics devices. Such devices lead the way to the electronic detection and coherent manipulation of SMMs spin states, important for quantum computation schemes at the single molecule level.

In this work, proposed recently [5], we realized a device consisting of SMMs anchored by supramolecular interactions on the sidewall of a chemical vapor deposition (CVD) grown carbon nanotube (CNT), itself connected to a three-terminal (transistor) geometry [6,7]. The CNT acts as a path for conducting electrons so that electronic transport does not occur directly through the magnetic orbitals of the SMM. This prevents charge-induced excitations or relaxation of molecular spin states. In particular, we showed that the electronic transport is extremely sensitive to the orientation of local magnetic moments. This property allows the electrical detection of magnetization reversal of individual molecular spins. In addition, a spin valve effect with two molecules leads to very large variations of the conductance with magnetoresistance ratios of up to several hundred percent. Our approach differs from previous realizations of carbon based spin valves [8-10] and does not imply 
magnetic leads. Indeed, the spin-dependent transport through this supramolecular spin valve is completely determined by the magnetic properties of the molecular species magnetically coupled to the conducting channel of the CNT. Similar results were recently obtained using graphene nanoconstriction decorated with $\mathrm{TbPc}_{2}$ magnetic molecules [11]. In this case, a magnetoconductivity signal as high as $20 \%$ was found for the spin reversal. These results show the behavior of multiple-field-effect nanotransistors with sensitivity at the single-molecule level.

The paper is structured as follows. In Section 2, we introduce different methods used to fabricate and measure the supramolecular based junctions. The results section (Section 3) describes the magnetic properties of the pyrene functionalized heteroleptic bis-phthalocyaninato-Terbium (III) SMMs (called $\mathrm{TbPc}_{2}$ in the following) used in this study. In Section 3.1, we briefly discuss the magnetization reversal mechanisms of the $\mathrm{TbPc}_{2} \mathrm{SMM}$, namely QTM and the direct relaxation process, using $\mu$-SQUID measurements on diluted crystals of molecules. Section 3.2 exhibits the electronic transport features of the spin valve, being in the closed quantum dot regime. Then, in Section 3.3, we present the spin-valve behavior revealed by the magneto-conductance measurements under magnetic field sweeps. The anisotropic dependence of the hysteretic conductance jumps is also studied and in good agreement with the expected uniaxial anisotropy of $\mathrm{TbPc}_{2} \mathrm{SMMs}_{\text {. Finally, Section }}$ 4 discusses the spin valve mechanism in further detail and we conclude with a brief outlook.

\section{Devices Fabrication}

In this project we studied about 150 samples of which 28 showed magnetic signals related to the $\mathrm{TbPc}_{2}$-SMMs and eight of them were studied in detail, manifesting similar behavior concerning their magneto-conductance. In contrast to our first publication [6], the sample presented in this paper was fabricated using CVD nanotubes. Catalyst islands were designed on $\mathrm{SiO}_{2}$ by creating holes by optical lithography in LOR3A resist, which were filled with $\mathrm{Fe} / \mathrm{Mo}$ catalyst in nanoporous alumina. After liftoff of the resist, nanotubes were grown in a Firstnano CVD oven at $750{ }^{\circ} \mathrm{C}$, whereby methane was used as a carbon source [12]. SWNTs were located by AFM or SEM with respect to lithographically patterned markers and then contacted with $50 \mathrm{~nm}$ Pd by standard electron-beam lithography, defining $200 \mathrm{~nm}$ long CNT junctions. A solution containing bis(phthalocyaninato)terbium (III) substituted with pyrene groups [13] with a $10^{-6}$ molarity was dropped on the device and dried under nitrogen flow. Samples with large resistance $(>100 \mathrm{k} \Omega$ ) at room temperature were micro bounded and measurements were carried out in a dilution fridge with a base temperature of $40 \mathrm{mK}$. The electronic temperature was estimated to be around $150 \mathrm{mK}$.

\section{Results}

\subsection{Magnetic Properties of the $\mathrm{TbP}_{2} S M M s$}

Among the existing SMMs families, single ion lanthanide complexes are among the most simple

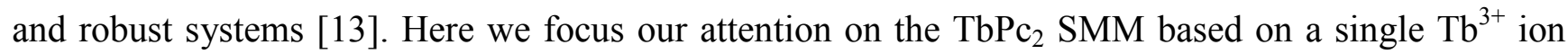
coordinated to two phthalocyanine (Pc) ligands as depicted in Fdigure 1(a). The SMM behavior originates from the electronic multiplet substructure of the $\mathrm{Tb}^{3+}$ ion in its $(4 \mathrm{f})^{8}$ electronic configuration leading to a $J=6$ magnetic moment. The $\mathrm{TbPc}_{2}-\mathrm{SMM}$ exhibits a significantly large axial magnetic 
anisotropy originating from the strong spin-orbit coupling in lanthanide ions and from the ligand field potential made by the two Pc ligands. It leads to a well-defined ground state configuration $\left(J=6,\left|J_{z}\right|=6\right)$ separated from the first excited state $\left(J=6, J_{\mathrm{z}}=5\right)$ by an energy splitting $\Delta E \sim 600 \mathrm{~K}$ [14]. All our measurements were performed at very low temperatures, so that we only consider the lowest energy substates available with $J_{z}= \pm 6$, the corresponding Zeeman diagram is plotted in Figure 1(b). Apart from the Terbium(III) ion, the molecule has a spin $1 / 2$ delocalized over the two phtalocyanines groups [15]. This unpaired electron mediates a magnetic coupling between the Terbium $4 \mathrm{f}$ electrons and its environment.

Figure 1. Magnetization reversal mechanisms in the $\mathrm{TbPc}_{2} \mathrm{SMM}$ (alkyl and pyrene substituents are omitted for reasons of clarity). (a) Scheme of the $\mathrm{TbPc}_{2}-\mathrm{SMM}$. The terbium ion has a $J=6$ magnetic moment and an unpaired electron is delocalized over the organic part; (b) Zeeman diagram calculated for the $\operatorname{TbPc}_{2} \operatorname{SMM}_{\text {ground state }}\left(J=6,\left|J_{z}\right|=6\right)$. The interaction with the $\mathrm{Tb}$ nucleus spin $I=3 / 2$ splits each electronic substate through the hyperfine coupling, providing a path for Quantum Tunneling of Magnetization (QTM) at the anti-crossing of two levels; (c) Hysteresis loops of the crystallized $\mathrm{TbPc}_{2}-\mathrm{SMM}(2 \%$ in the $\mathrm{YPc}_{2}$ matrix) measured at $40 \mathrm{mK}$ for different sweeping rates ranging from 1 to $280 \mathrm{mT} . \mathrm{s}^{-1}$. QTM reflects in staircase-like steps of the hysteresis loops at low magnetic fields, each step corresponding to a level anti-crossing. Molecules, which did not undergo QTM can relax their magnetization to a lower energy state by the direct transition (DT) occurring at larger magnetic fields.

(a)

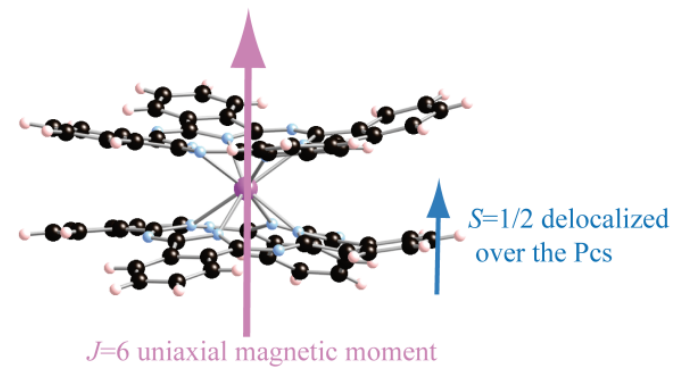

(b)

(c)

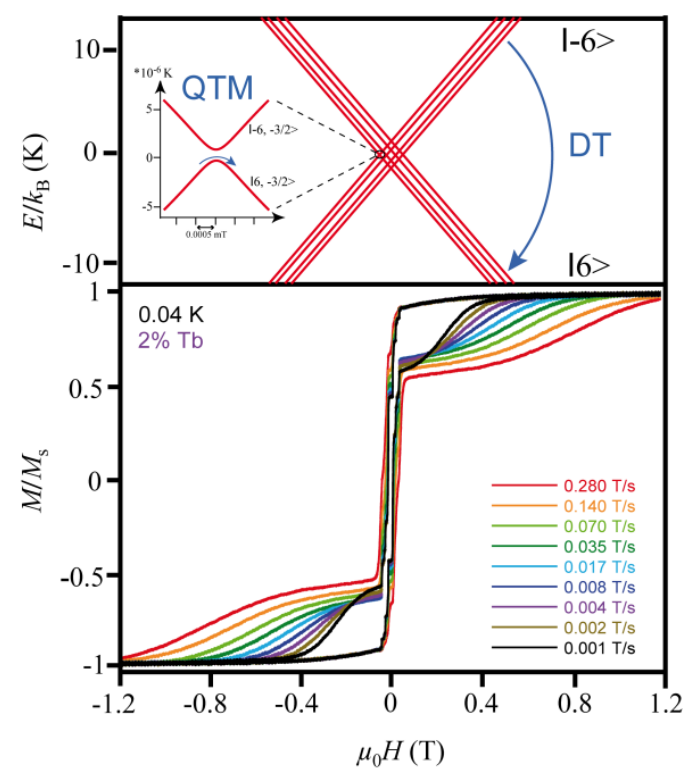


Figure 1(c) presents $\mu$-SQUID magnetization hysteresis loops of the $\mathrm{TbPc}_{2} \mathrm{SMMs}$ at a temperature of $40 \mathrm{mK}$. The SMMs have been diluted in an $\mathrm{YPc}_{2}$ paramagnetic matrix to minimize any magnetic intermolecular interactions. At an applied magnetic field of $-1.2 \mathrm{~T}$ along the easy-axis, all magnetic moments in the crystal are saturated along the same direction. Around zero-field, a large number of these magnetic moments switch their magnetization orientation through staircase-like steps of the hysteresis loop. The origin of these magnetization steps is the QTM between the $J_{\mathrm{z}}=6$ and $J_{\mathrm{z}}=-6$ substates. Since the other excited substates are, energy-wise, far above the fundamental ones, transitions involving the excited states cannot occur. As a consequence, the origin of QTM in single ion rare earth based SMMs differs strongly from the 3d-metal-cluster SMM cases. This comes from the interaction of the $\mathrm{Tb}$ ion with the ligand field. Besides this, the $\mathrm{Tb}$ nucleus owns a nuclear spin of $I$ $=3 / 2$ with a natural isotopic abundance of $100 \%$. The strong hyperfine coupling in $\mathrm{TbPc}_{2}$ leads to a splitting of the $J=6$ electronic multiplet in several $\left|J_{Z}\right\rangle \mid I_{Z}>$ coupled states. These states are visible in the Zeeman diagram in Figure 1(b). QTM can occur through appropriate magnetic field conditions where two such states are brought to resonance at an avoided crossing of two levels (Figure 1(b)). The remaining magnetic moments, which did not undergo magnetization reversal by QTM around the zero magnetic field, start to reverse at about a few hundreds of $\mathrm{mT}$. This transition is visible in the hysteresis loops in Figure 1(c) as a broad field scan rate dependent step. This one phonon mediated mechanism, called the direct transition (DT), is schematized in Figure 1(b).

We highlight that those measurements have been carried out on an assembly of $\mathrm{TbPc}_{2}-\mathrm{SMMs}_{\mathrm{s}} \mathrm{a}$ crystallized form. One could thus wonder whether the SMM properties may still be observed on sub-monolayers or isolated molecules deposited on a surface. Indeed, SMMs may lose their magnetic properties when attached to metallic surfaces, as was shown e.g., for $\mathrm{Mn}_{12}$-acetate [16]. A slight modification of the ions surroundings can lead to a drastic change of the crystal field potential and thus to an alteration of the magnetic properties [17]. However, in the case of the $\mathrm{TbPc}_{2}-\mathrm{SMM}$, it has been recently demonstrated that the structural and magnetic properties are still conserved [18]. The main fingerprints of SMMs, that is, QTM around zero magnetic field, strong axial anisotropy and high superparamagnetic blocking temperature are, indeed, still present. The $\mathrm{TbPc}_{2}-\mathrm{SMM}$ is one of the most interesting and reliable systems in order to study magnetism at single molecular level. Besides, this molecule is very well suited to be attached to $\mathrm{sp}^{2}$ carbon nanomaterials, such as carbon nanotubes or grapheme [19] via supramolecular $\pi-\pi$ interactions. This strategy has been used to build our supramolecular spin valve and is described in the following.

\subsection{Electronic Transport through Carbon Nanotubes Functionalized by $\mathrm{TbPc}_{2}-\mathrm{SMMS}$}

The original geometry of our devices is presented in Figure 2(a). A CNT, contacted with non-magnetic electrodes, forms a quantum dot (QD), which is laterally coupled to several $\mathrm{TbPc}_{2}-\mathrm{SMMs}_{\mathrm{s}}$ through $\pi-\pi$ stacking interaction. Indeed, phtalocyanine groups can be functionalized with pyrenes ligands, allowing a supra-molecular anchoring point and a better coupling to $\pi$-conjugated systems such as carbon nanotubes [20]. 
Figure 2. Electronic transport in carbon nanotube quantum dots with grafted $\mathrm{TbPc}_{2}$ molecules. (a) Artist view of the device scheme, consisting of an electrically connected carbon nanotube junction, laterally coupled to isolated $\mathrm{TbPc}_{2}-\mathrm{SMMs}$; (b) Color-scale plots of the differential conductance $d I / d V$ at temperature $T=40 \mathrm{mK}$, as a function of source-drain voltage $V_{s d}$ and back-gate voltage $V_{g}$, displaying the charge stability diagram in the Coulomb blockade regime. The typical charging energy is about $20 \mathrm{meV}$; (c-d) Enlarged views of (b), showing the charge degeneracy point around $V_{g}=1.79 \mathrm{~V}$ at constant static magnetic fields (c) $\mu_{0} H=0 \mathrm{~T}$ and (d) $\mu_{0} H=1 \mathrm{~T}$.

(a)

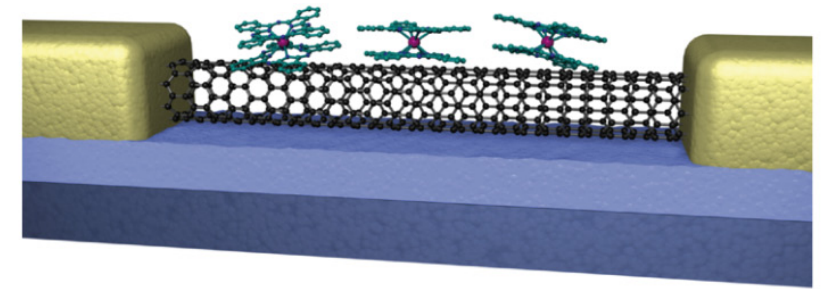

(b)

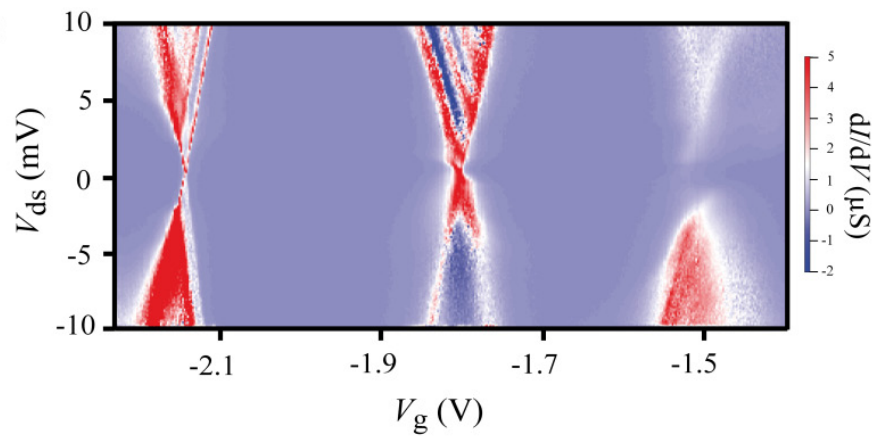

(c)

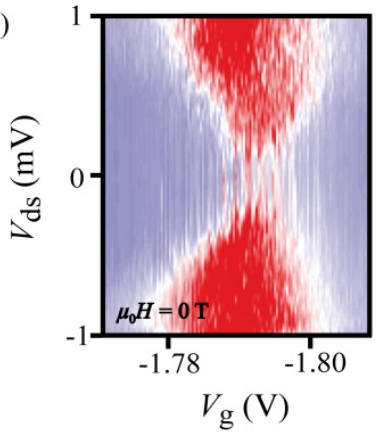

(d)

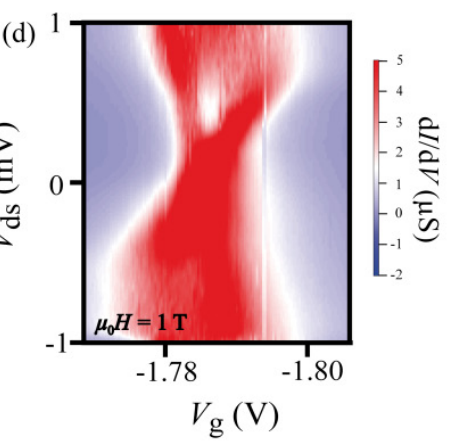

When deposited, the $\mathrm{TbPc}_{2}-\mathrm{SMM}$ anchors on the nanotube in a way that the substituted Pc ligand of the molecule comes directly in contact with the nanotube surface. The energy gain through supramolecular interactions is maximized by the formation of strong aromatic $\pi-\pi$ and $\mathrm{C}-\mathrm{H}-\pi$ stacking interactions between the substituted Pc and CNT. Because of the efficient hybridization between Pc and CNT orbitals [21], we can assume that the unpaired electron delocalized over the Pc ligands has strong interaction with the conduction electron of the nanotube.

The supra-molecular device structure is cooled down in a ${ }^{3} \mathrm{He} /{ }^{4} \mathrm{He}$ dilution refrigerator to $40 \mathrm{mK}$ and a differential conductance measurement is conducted with an Adwin real-time acquisition system, programmed in a lock-in mode. The lock-in amplitude and frequency are set to $50 \mu \mathrm{V}$ and $33 \mathrm{~Hz}$, respectively. The QD characteristics are measured by bias spectroscopy, that is, the differential conductance $\mathrm{d} I / \mathrm{d} V$ is plotted in a color code as a function of the back-gate and bias voltages. 
Figure 2(b) shows the standard Coulomb diamond expected for a CNT QD with a charging energy around $20 \mathrm{meV}$. Figures $2(\mathrm{c}, \mathrm{d})$ display a zoom on the degeneracy points without and under magnetic field (1 T). At low bias $(<1 \mathrm{mV})$ and without magnetic field (Figure 2(c)), the degeneracy point has a noisy conductance, which fluctuates between two values. Then under $1 \mathrm{~T}$, the conductance becomes stable and closed (Figure 2(d)). These features reveal the presence of an extra tunnel barrier in the QD [22], which can be modulated by the magnetic field.

\subsection{Characterization of the Strongly Anisotropic Spin-Valve Effect}

The magnetoresistance measurements, at the previously mentioned degeneracy point (see Figure 2), are presented in Figure 3(a). At $-1 \mathrm{~T}$, the differential conductance is saturated to its maximum value. Sweeping up the magnetic field at $20 \mathrm{mT} / \mathrm{s}$ until zero-field, $\mathrm{d} / / \mathrm{d} V$ drops down abruptly to its minimum value. When still increasing the field, $\mathrm{d} I / \mathrm{d} V$ abruptly recovers its original value. The complete measurement from $-1 \mathrm{~T}$ to $+1 \mathrm{~T}$ (trace) and back to $-1 \mathrm{~T}$ (retrace) forms a hysteresis loop, which is characteristic of a spin-valve device.

Figure 3. Conductance hysteresis loops of the supramolecular spin valve. (a-b) Differential conductance $d I / d V$ measured at $T=40 \mathrm{mK}$ as a function of in-plane magnetic field $\mu_{0} H$ applied respectively along (a) the easy axis direction $\left(0^{\circ}\right)$; and (b) the hard direction $\left(90^{\circ}\right)$ of magnetization. The red and blue arrows indicate the magnetic field sweep directions; (c) Color-scale plot of the $d I / d V$ hysteresis (obtained from the difference between both field sweep directions) as a function of the applied magnetic field angle. The white color code is associated to zero hysteresis (reproducible $d I / d V$ curves); (d) Relative disposition of the molecule with respect to the nanotube. The magnetic hard axis is $30^{\circ}$ tilted from the nanotube axis.
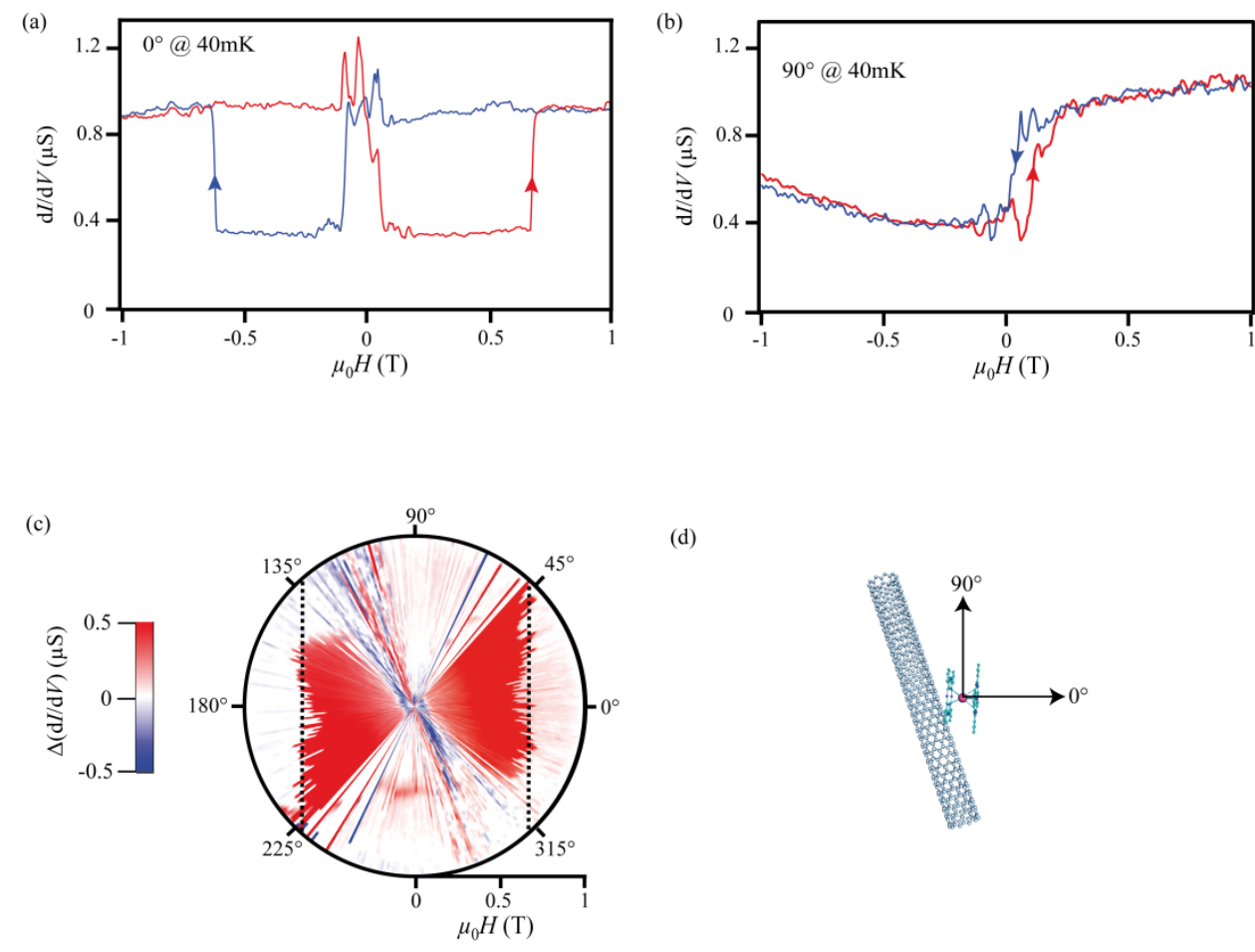

(d)

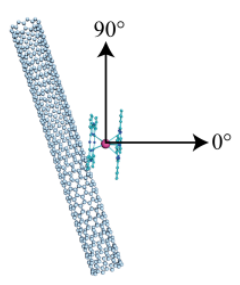


Another remarkable feature of our device is its anisotropic response: we observed that the field values at which sharp conductance jumps are measured, called switching fields, depend strongly on the direction of the applied field. From a certain angle, our magnetic field is not strong enough to observe switching, and the hysteresis disappears (Figure 3(b)). In this case, the differential conductance does not depend on the field history; $\mathrm{d} I / \mathrm{d} V$ is minimum for negative magnetic field and maximum for positive magnetic field, demonstrating that the switching near zero-field occurs independently from the one taking place at larger field. Thereby, we have to consider several independent magnetic objects to explain the data.

In order to go one step further, we have plotted on Figure 3(c) the hysteresis amplitude (difference between trace and retrace) as a function of the applied field direction. It turns out that the switching field occurring at high value (the dashed line on Figure 3(c)) describes a straight line in the field space. The projection of those points along one axis stays constant, which is the fingerprint of the Ising like uniaxial anisotropy of the $\mathrm{TbPc}_{2}$-SMM family.

Figure 4(a) shows the dependence of the hysteresis on the bias voltage applied to the quantum dot. This bias dependence shows that the hysteresis persists until $1 \mathrm{mV}$, but above this value, any residual hysteresis is smaller than the noise level. Furthermore, this measurement provides additional information: the switching field evolves with the bias voltage. We conclude from this observation that the conduction electrons can excite the molecules for larger bias voltages. Indeed, the energy of the conduction electrons increases with the bias voltage. A part of this energy might be transferred to the molecule via the electron density on the Pc-ligands and therefore to the anisotropic $\mathrm{Tb}$ spin system.

As the temperature increases, the amplitude of the magnetoresistance decreases (Figure 4(b)). Indeed the spin-phonon interaction increases with the temperature and affect the spin-coherence. The

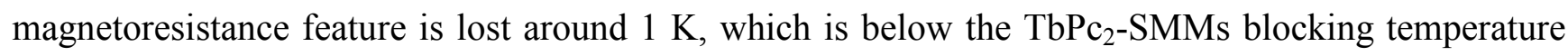
but is in agreement with an exchange interaction of a few hundreds of $\mu \mathrm{V}$. The blocking temperature can be roughly estimated to be around $1.5 \mathrm{~K}$, in agreement with recent XMCD measurements of $\mathrm{TbPc}_{2}$-SMMs monolayers [18].

Figure 4. Bias and temperature dependences of the conductance hysteresis loops: (a) Color scale map of the $d I / d V$ hysteresis as a function of in-plane magnetic field and source drain voltage $V_{d s}$. The magnetic hysteresis are suppressed above $V_{d s}= \pm 1 \mathrm{mV}$; (b) 15 records of conductance hysteresis loops for several temperatures ranging from 0.04 to $1 \mathrm{~K}$ at a constant sweep rate of $50 \mathrm{mT} / \mathrm{s}$. The curves for $T>40 \mathrm{mK}$ are offset by a multiple of 200 $\mathrm{nS}$ for clarity.

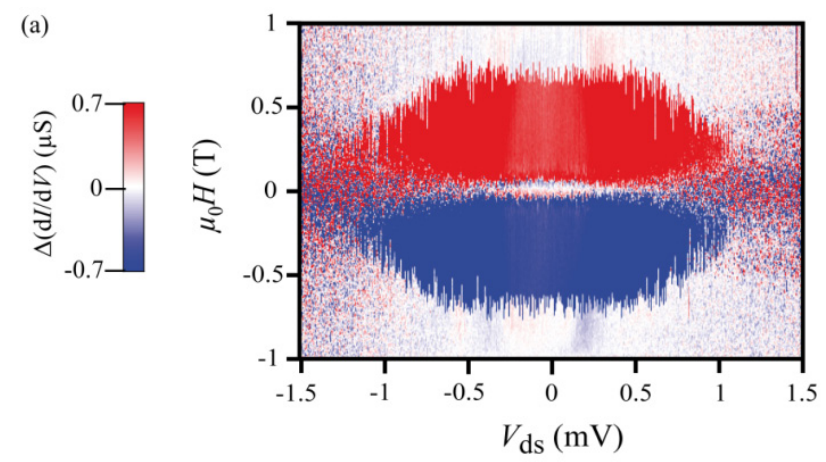


Figure 4. Cont.

(b)

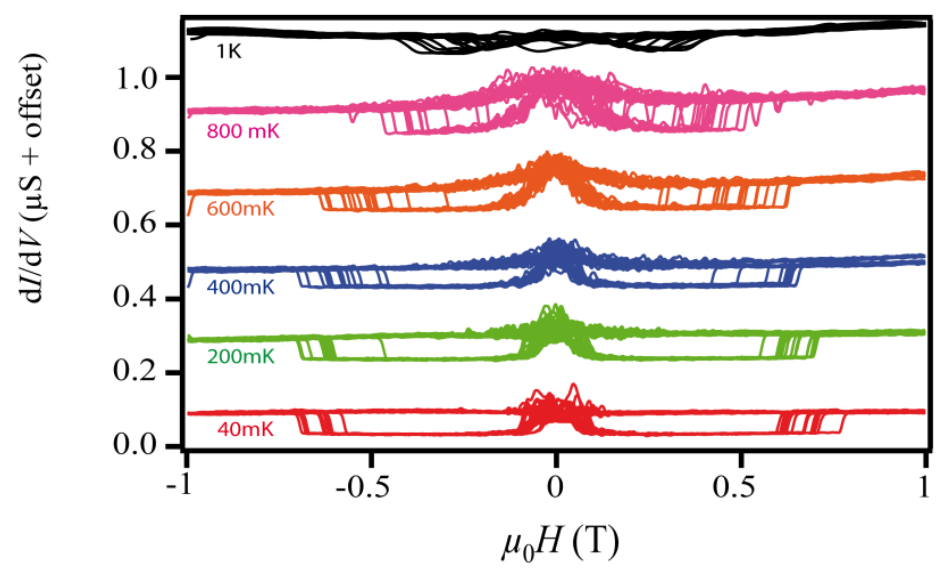

\section{Discussion}

The spin-valve features can be explained by a simple model involving two distinct molecules: one with a close to one QTM probability and another one only subjected to DT. Under a high magnetic field, the magnetic moments of both molecules are polarized in parallel. This situation corresponds to the high conductance regime. When the magnetic field is reduced, one of the molecule experiences QTM close to zero field, whereas the other stays in the same state. The device is then in an antiparallel configuration and the conductance is minimum. Finally, when the following switching field is reached, meaning when the second molecule undergoes a DT, the parallel arrangement, and therefore the original conductance value, are recovered. This is, of course, the idealized case. In fact, by looking closely at the QTM region, we can observe several abrupt changes of conductance. It means that several molecules (more than two) are involved in the close to zero field process. However, only one molecule relaxes at larger fields via a DT.

From a microscopic point of view, each of these molecules interacts with the nanotube by creating a localized spin-polarized state in its vicinity (schematized in Figure 5(a)). A dipolar interaction is not sufficient to explain such an effect. Indeed, the dipolar interaction between the $S=1 / 2$ radical on the Pcs and the conduction electrons cannot exceed a few tens of $\mathrm{mT}\left(1 / 2 \mu_{\mathrm{B}}\right.$ creates a $1 / 2 \mathrm{~T}$ dipolar field at $1 \AA$, and $\sim 20 \mathrm{mT}$ at $3 \AA$ ). Also, even if we consider the effect of the moment $J=6$ on the conduction electron, the dipolar field is of the same order of magnitude, which is too small to explain the strength of the effect. Considering an exchange interaction, mediated by the $\pi$-electron density in the organic Pc ligand, seems to be more realistic. Indeed, $\mathrm{Hu}$ et al. [23] have shown that spin-polarization may occur through the interaction between a $\pi$-system delocalized over a carbon chain and latterly coupled spin radical. As shown by Gambardella et al. [24] in a similar system, the electron density on the Pc ligand is able to mediate the magnetic information by a strong exchange interaction. The molecules induce localized states on the nanotube by lifting the spin degeneracy through this interaction (Figure 5(b)). The strength of this interaction can be estimated to be around $200 \mu \mathrm{V}$ by looking at the gapped degeneracy point in Figure 2(d). The spin level splitting is inhomogeneous along the tube when both molecules are in the antiparallel configuration, as a result of the mismatch between energy levels for a same spin (Figure 5(c)). This barrier can be overcome by 
applying a bias voltage higher than the exchange interaction between the molecule and the conduction electrons.

Figure 5. (a) Scheme of the localized dots induced by hybridization between the molecules and the nanotube; (b) Both molecules are polarized in the same manner. It induces a Zeeman splitting identical for both sets of localized states; (c) In the antiparallel configuration the Zeeman splitting is inhomogeneous, preventing spin transport through the device, unless a bias higher than the exchange interaction is applied.

(a)

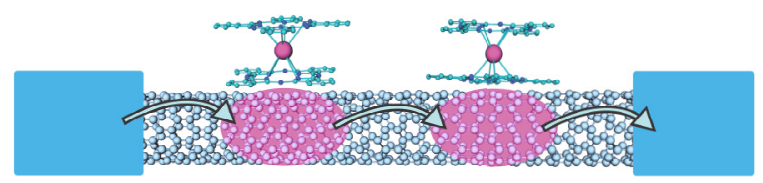

(b)

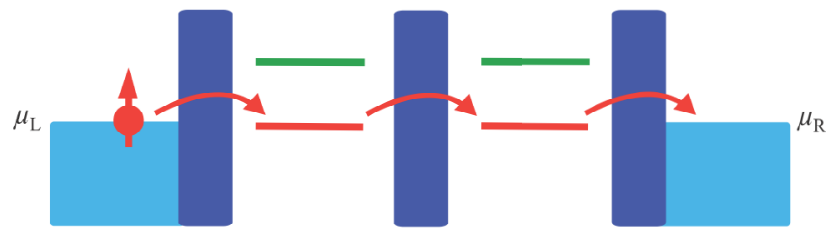

(c)

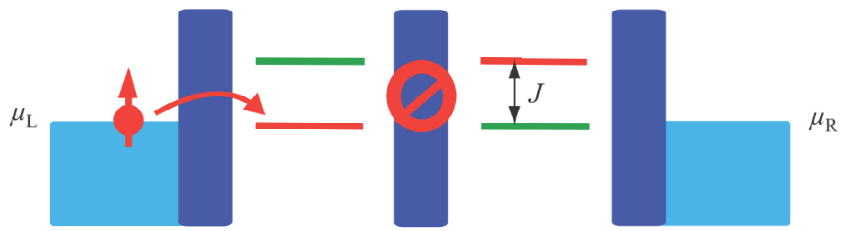

\section{Conclusions}

We have reported on the characterization of a fully molecular spin-valve made of a carbon nanotube laterally coupled to a few single-molecule magnets determining for the first time the strength of the exchange energy between the SMMs. The interaction between both objects is strong enough to allow an abrupt modification of the conductance by changing the magnetization direction of only one molecule. As a result, the device exhibits a spin-valve effect and the magneto-conductance ratio can reach a few hundreds of percents. These features are obviously related to the grafted TbPc2-SMMs. Indeed, the anisotropic response clearly corresponds to an Ising-like uniaxial magnetic system. Our results open a pathway toward the fabrication of an all-organic spintronic device by the use of supramolecular self-organization techniques. Moreover, the high sensitivity of the device allows the characterization and the control of a single localized spin. Thus, an entanglement between different spin systems could also be possible using the CNT as tunable bus.

\section{Acknowledgments}

This work is partially supported by the DFG programmes SPP 1459 and TRR 88 "3Met", ANR-PNANO project MolNanoSpin No ANR-08-NANO-002, ERC Advanced Grant MolNanoSpin No 226558, STEP MolSpinQIP and the Nanosciences Foundation of Grenoble. Samples were 
fabricated in the NANOFAB facility of the Néel Institute and we thank B. Fernandez, G. Julie, T. Crozes and T. Fournier for help in device fabrication. We thank M. Affronte, F. Balestro, N. Bendiab, L. Bogani, E. Bonet, V. Bouchiat, L. Calvet, A. Candini, D. Feinberg, J. Jarvinen, L. Marty, R. Maurand, T. Novotny, R. Piquerel, C. Thirion and R. Vincent for fruitful discussions and software development. We thank E. Eyraud, R. Haettel, C. Hoarau, D. Lepoittevin and V. Reita for technical support.

\section{References}

1. Christou, G.; Gatteschi, D.; Hendrickson, D.N.; Sessoli, R. Single-molecule magnets. MRS Bull. 2000, 25, 66-71.

2. Wernsdorfer, W.; Sessoli, R. Quantum phase interference and parity effects in magnetic molecular clusters. Science 1999, 284, 133-135.

3. Xiong, Z.H.; Wu, D.; Vardeny, Z.V.; Shi, J. Giant magnetoresistance in organic spin-valves. Nature 2004, 427, 821-824.

4. Barraud, C.; Seneor, P.; Mattana, R.; Fusil, S.; Bouzehouane, K.; Deranlot, C.; Graziosi, P.; Hueso, L.; Bergenti, I.; Dediu, V.; Petroff, F.; Fert, A.. Unravelling the role of the interface for spin injection into organic semiconductors. Nat. Phys. 2010, 6, 615-620.

5. Bogani, L.; Wernsdorfer, W. Molecular spintronics using single-molecule magnets. Nat. Mater. 2008, 7, 179-184.

6. Urdampilleta, M.; Klyatskaya, S.; Cleuziou, J.-P.; Ruben, M.; Wernsdorfer, W. Supramolecular spin valves. Nat. Mater. 2011, 10, 502-506.

7. Sanvito, S. Filtering spins with molecules. Nat. Mater. 2011, 10, 484-485.

8. Hueso, L.E.; Pruneda, J.M.; Ferrari, V.; Burnell, G.; Valdés-Herrera, J.P.; Simons, B.D.; Littlewood, P.B.; Artacho, E.; Fert, A.; Mathur, N.D. Transformation of spin information into large electrical signals using carbon nanotubes. Nature 2007, 445, 410-413.

9. Aurich, H.; Baumgartner, A.; Freitag, F.; Eichler, A.; Trbovic, J.; Schönenberger, C. Permalloybased carbon nanotube spin-valve. Appl. Phys. Lett. 2010, 97, 153116.

10. Kim, W.Y.; Kim, K.S. Prediction of very large values of magnetoresistance in a graphene nanoribbon device. Nat. Nanotechnol. 2008, 3, 408-412.

11. Candini, A.; Klyatskaya, S.; Ruben, M.; Wernsdorfer, W.; Affronte, M. Graphene spintronic devices with molecular nanomagnets. Nano Lett. 2011, 11, 2634-2639.

12. Kong, J.; Soh, H.T.; Cassell, A.M.; Quate, C.F.; Dai, H. Synthesis of individual single-walled carbon nanotubes on patterned silicon wafers. Nature 1998, 395, 1-4.

13. Klyatskaya, S.; Galan-Mascarós, J.R.G.; Bogani, L.; Hennrich, F.; Kappes, M.; Wernsdorfer, W.; Ruben, M. Anchoring of rare-earth-based single-molecule magnets on single-walled carbon nanotubes. J. Am. Chem. Soc. 2009, 131, 15143-15151.

14. Ishikawa, N.; Sugita, M.; Wernsdorfer, W. Quantum tunnelling of magnetization in lanthanide single-molecule magnets: bis(phthalocyaninato)terbium and bis(phthalocyaninato)dysprosium anions. Angew. Chem. Int. Ed. 2005, 44, 2931-2935.

15. Ishikawa, N.; Sugita, M.; Okubo, T.; Tanaka, N.; Iino, T.; Kaizu, Y. Determination of ligand-field parameters and f-electronic structures of double-decker bis(phthalocyaninato)lanthanide complexes. Inorg. Chem. 2003, 42, 2440-2446. 
16. Ishikawa, N.; Sugita, M.; Tanaka, N.; Ishikawa, T.; Koshihara, S.-ya.; Kaizu, Y. Upward temperature shift of the intrinsic phase lag of the magnetization of Bis(phthalocyaninato)terbium by ligand oxidation creating an $\mathrm{S}=1 / 2$ spin. Inorg. Chem. 2004, 43, 5498-5500.

17. Mannini, M.; Sainctavit, P.; Sessoli, R.; Moulin, C.C.D.; Pineider, F.; Arrio, M.A.; Cornia, A.; Gatteschi, D. XAS and XMCD investigation of Mn-12 monolayers on gold. Chem. Eur. J. 2008, $14,7530-7535$.

18. Sorace, L.; Benelli, C.; Gatteschi, D. Lanthanides in molecular magnetism: Old tools in a new field. Chem. Soc. Rev. 2011, 40, 3092-3104.

19. Margheriti, L.; Chiappe, D.; Mannini, M.; Car, P.-E.; Sainctavit, P.; Arrio, M.-A.; Buatier de Mongeot, F.; Cezar, J.; Piras, F.; Magnani, A.; et al. X-Ray detected magnetic hysteresis of thermally evaporated terbium double-decker oriented films. Adv. Mater. 2010, 22, 5488-5493.

20. Lopes, M.; Candini, A.; Urdampilleta, M.; Reserbat-Plantey, A.; Bellini, V.; Klyatskaya, S.; Marty, L.; Ruben, M.; Affronte, M.; Wernsdorfer, W.; et al. Surface-enhanced Raman signal for terbium single-molecule magnets grafted on graphene. ACS Nano 2010, 4, 7531-7537.

21. Wang, Y.; Hu, N.; Zhou, Z.; Xu, D.; Wang, Z.; Yang, Z.; Wei, H.; Kong, E.S.-W.; Zhang, Y. Single-walled carbon nanotube/cobalt phthalocyanine derivative hybrid material: preparation, characterization and its gas sensing properties. J. Mater. Chem. 2011, 21, 3779-3787.

22. Bockrath, M.; Liang, W.; Bozovic, D.; Hafner, J.H.; Lieber, C.M.; Tinkham, M.; Park, H. Resonant electron scattering by defects in single-walled carbon. Science 2001, 291, 283-285.

23. Hu, G.; Guo, Y.; Wei, J.; Xie, S. Spin filtering through a metal/organic-ferromagnet/metal structure. Phys. Rev. B 2007, 75, 1-6.

24. Lodi Rizzini, A.; Krull, C.; Balashov T.; Kavich, J.; Mugarza, A.; Miedema, P.; Thakur, P.K.; Sessi, V.; Ruben, M.; Stepanow, S.; Gambardella, P. Coupling single-molecule magnets to ferromagnetic substrates. Phys. Rev. Lett. 2011, in press.

(C) 2011 by the authors; licensee MDPI, Basel, Switzerland. This article is an open access article distributed under the terms and conditions of the Creative Commons Attribution license (http://creativecommons.org/licenses/by/3.0/). 